\title{
Similarity: generalizations, applications and open problems
}

\author{
G. Bluman · P. Broadbridge · J. R. King • \\ M. J. Ward
}

Received: 24 August 2009 / Accepted: 2 September 2009 / Published online: 30 October 2009

(C) Springer Science+Business Media B.V 2009

\section{Introduction}

This Special Issue of the Journal of Engineering Mathematics focuses on recent theoretical and applied developments in Similarity, including asymptotic/numerical similarity and associated software. In particular, it is devoted to papers by leading researchers who apply similarity analysis to engineering and biological problems (asymptotics involving similarity solutions), develop significant extensions of similarity methods to find and use symmetries and conservation laws of partial differential equations (PDEs), develop and use numerical methods for PDEs based on admitted symmetries and/or conservation laws, and develop symbolic manipulation software to implement similarity analyses. As a consequence, it is hoped that these four distinct groups of similarity researchers will more readily become aware of recent developments and needs in related fields. In turn, this should lead to important and fruitful research directions. These papers arose from presentations at a conference on Similarity: Generalizations, Applications and Open Problems in Vancouver, British Columbia, August 11-15, 2009, sponsored by PIMS (Pacific Institute for the Mathematical Sciences) and AMSI (Australian Mathematical Sciences Institute). This introductory article includes a brief summary of the papers as well as a reference to part of the discussion on open problems during a session of the conference.

G. Bluman $(\varangle) \cdot$ M. J. Ward

Department of Mathematics, University of British Columbia, Vancouver, BC V6T 1Z2, Canada e-mail: bluman@math.ubc.ca

M. J. Ward

e-mail: ward@math.ubc.ca

P. Broadbridge

School of Engineering and Mathematical Sciences, La Trobe University, Bundoora, VIC 3086, Australia

e-mail: P.Broadbridge@latrobe.edu.au

J. R. King

School of Mathematical Sciences, University of Nottingham, Nottingham NG7 2RD, UK

e-mail: john.king@nottingham.ac.uk 


\subsection{Similarity}

Similarity is concerned with invariance of an aspect of a given problem under continuous symmetries. When a problem is invariant under a continuous transformation, i.e., it has a continuous symmetry, then often the complexity in solving it is reduced by the dimension of the symmetry. A natural scaling invariance of a problem arises from dimensional consistency (dimensional analysis). This is fundamental to modelling (cf. [1-11]). More generally, similarity (symmetry) analysis has become a very sophisticated subject. For a given problem, often one can determine admitted symmetries and conservation laws that are not obvious by inspection. In turn, one can use admitted symmetries and conservation laws for intermediate or long-time asymptotic analysis, as an aid for determining numerical solutions, to obtain particular solutions, to map the problem to a simpler problem (e.g., linearization), etc.

Self-similar solutions for PDEs arise for many real problems from invariance under dimensional analysis. More generally, self-similar solutions arise from invariance under scalings of variables.

When the form of the similarity reduction can be identified a priori (often by means of a conservation law), the result is often termed a self-similar solution of the first kind; when the similarity exponent must be determined as the eigenvalue in an eigenvalue problem for the similarity solution, the latter is termed "second kind" (the most widely studied such examples involving the calculation of travelling-wave speeds).

Still more generally, if a PDE system is invariant under a Lie group of point transformations (point symmetries), one can find, constructively, similarity solutions (invariant solutions) that are invariant under a subgroup of the full group admitted by the PDE system. Similarity solutions arise from solving a reduced system of DEs with fewer independent variables. Similarity solutions can be constructed for specific boundary value problems.

Beginning in the 1980s, and continuing to date, there have been numerous studies analyzing finite-time singularity formation for nonlinear parabolic second-order PDEs (cf. [12-17]) and more recently for their higherorder generalizations (cf. [18-20]). The applications of singularity formation are diverse, including fourth-order lubrication theory models from fluids (cf. [21-23]), chemotactic aggregation governed by systems of nonlinear PDEs (cf. [24,25]), and quenching behaviour in a nonlinear PDE model of a MEMS capacitor (cf. [26]). For such problems, a singularity forms in finite time whereby either the solution or its partial derivatives diverge. Although generally it is not possible to determine analytically the singularity time or point(s) where a solution loses its regularity, it is of considerable interest to characterize analytically the local solution (typically a similarity solution) behaviour near a singularity, and to determine the stability of any locally self-similar solutions. Therefore, in this context, similarity solutions of such PDEs describe local solution behaviour near singularities. A comprehensive survey of rigorous, formal, and numerical approaches to local self-similarity in describing singularity behaviour in a wide class of physically based PDE models, relating primarily to fluid systems, is given in [27]. An extensive bibliography of further problems is given in the bibliography of [28].

Similarity solutions also play an essential role in describing intermediate-asymptotic behaviour for wide classes of PDEs (cf. [5,7]). In particular, important issues arising from the study of similarity solutions include:

- rigorous stability properties of local self-similar solutions for second-order PDE models characterizing singularity formation is rather well-developed (cf. [16,29]) owing to the Sturm-Liouville structure of the linearized operator, leading to a countably discrete spectrum. In contrast, for high-order PDE models exhibiting singularity formation, there can be a countably infinite set of local self-similar solutions whose stability properties must be studied on a case-by-case basis (cf. [18-20,27]);

- the role of quasi-self-similar solutions (which satisfy the governing equations only asymptotically, rather than exactly) in describing ultimate (long-time or close to finite-time singularity) evolutions (cf. [17,30-32]). Remarkably, such solutions arise often in applications (cf. [27,28]) and their analysis requires a synthesis of similarity, dynamical systems, perturbation, and numerical methods;

- rigorous analysis of the above issues (cf. [12-17,29]). In many cases such analysis builds directly on the formal results; 
- the development of systematic symmetry-based techniques for identifying candidate intermediate-asymptotic solutions (see, for example, [7]; there is considerable further scope for the application of modern symmetry approaches).

In recent years there have been many developments in extending symmetry-based methods to find similarity solutions and conservation laws for PDE systems. Such advances have focused on how to:

- find and implement further applications for admitted symmetries (cf. [10,11,33-36]);

- find multipliers yielding conservation laws and the resulting fluxes of conservation laws (cf. [37-42]);

- extend the spaces of admitted symmetries and hence construct further similarity solutions (cf. [10,11,33,42-47]);

- extend the applications to construct solutions from admitted symmetries to include admitted "symmetries" arising from generalizations (similarity solutions arising from the nonclassical and other related methods) (cf. $[10,42,48-55])$;

- efficiently solve the (overdetermined) linear systems of determining equations for symmetries or conservation law multipliers and solve the nonlinear systems of determining equations for the nonclassical and related methods through the development of symbolic manipulation software (cf. [42,56-71]);

- develop numerical schemes that effectively use symmetries and/or conservation laws for ODE's (cf. [72]), for difference equations (cf. [73,74]), and for PDE models that exhibit finite-time singular behaviour (cf. [75-77]).

\section{Contents of this issue}

The articles in this issue can be grouped into the following rough classification.

\subsection{Self-similarity}

As mentioned previously, self-similar solutions arise naturally as particular solutions of PDE systems from dimensional analysis and, more generally, from invariance of PDE systems under scalings of variables. Usually, such solutions do not globally satisfy imposed boundary conditions. However, through delicate analysis, one can often show that a self-similar solution holds asymptotically in certain identified domains (near singularities, large time, small time, intermediate, etc).

\subsubsection{Stability and dynamics of self-similarity in evolution equations}

In [28], a methodology is presented for studying linear stability for self-similar solutions. It is shown that self-similar phenomena can be studied through use of many ideas arising in the study of dynamical systems. In particular, there is a discussion of the role of symmetries in the context of stability of self-similar dynamics. For blow-up solutions, it is demonstrated that symmetries give rise to positive eigenvalues associated with rescaling symmetries as opposed to instability. Moreover, it is shown how such a stability analysis can identify a unique (and observable) stable solution from a countable infinity of self-similar solutions. It is argued that linearization methods, combined with careful analysis of associated symmetries, provide a powerful tool for analyzing stability of self-similar solutions.

\subsubsection{Thin-film rupture for large slip}

In [78], there is a study of the rupture of thin liquid films on hydrophobic substrates, assuming large slip at the liquid/solid interface. Using a strong-slip lubrication model, it is shown that the rupture can pass through up to three self-similar regimes with different dominant balances and different scaling exponents. For one of the regimes, there is a self-similar solution of the second kind, and the similarity (scaling) exponent is determined by solving a boundary-value problem for a corresponding reduced nonlinear ODE. Moreover, in this regime it is shown that the self-similar solution blows up after a finite time. 


\subsubsection{Self-similarity in particle-laden flows at constant volume}

In [79], there is consideration of constant volume thin-film slurries on an incline. Clear fluids in this geometry are known to have a front position that moves according to a $t^{1 / 3}$ scaling law, based on self-similar solution analysis [80]. By comparing theory with experiments, it is shown that the $t^{1 / 3}$ scaling law persists, to leading order, for slurry flows with particle settling.

\subsubsection{Asymptotic analysis of extinction behaviour in fast nonlinear diffusion}

In [81], there is a summary of the range of applications that arise for the equation of fast nonlinear diffusion. There is a discussion of relevant similarity solutions and intermediate-asymptotic behaviour arising from the invariance of the fast nonlinear diffusion equations under translations in space and time and a two-parameter family of scalings. In turn, this yields a one-parameter family of self-similar solutions for consideration to obtain relevant asymptotic solutions (in terms of determining appropriate values in different regimes for the free scaling parameter) through delicate analysis in terms of posed data.

\subsection{Applications of symmetry methods}

In the past, symmetries and conservation laws used in applications and the uses themselves were often obvious by inspectional analysis. However, in recent years, with the development of more sophisticated symmetry methods and especially with the help of ever-improving software, one is able to find nontrivial uses of symmetries and conservation laws in applications.

\subsubsection{Temperature-dependent surface diffusion near a grain boundary}

For a linear partial differential equation, an admitted point symmetry (Lie symmetry) leads algorithmically, through a corresponding separation of variables, to solutions that are much more general than the usual similarity solutions directly obtained by symmetry reduction. This allows one to formally solve free-boundary problems that have approximate symmetries at early times. In [82], this method is applied to a complicated practical boundary-value problem for fourth-order surface diffusion near a grain boundary at changing temperature.

\subsubsection{Relevance of symmetry methods in mechanics of materials}

In [83], the interest and relevance of symmetry methods as a predictive and systematic methodology in the continuum mechanics of materials are analyzed, relying on a classification of the inherent aspects in terms of direct, extended direct and inverse methods. In particular, the direct problem of finding invariants associated with a given material's constitutive law, including dissipation is considered as well as the inverse problem of constructing a material's constitutive law from invariance under a given Lie group of transformations.

\subsubsection{Higher-order symmetries and conservation laws of the G-equation for premixed combustion and resulting numerical schemes}

In [84], it is shown that the set of computable local symmetries of the $G$-equation for flame front propagation of premixed combustion is considerably extended if higher-order symmetries are considered. In particular, it is shown that the $G$-equation admits an infinite number of higher-order symmetries for an arbitrary velocity field. Geometrical and kinematic interpretations of the symmetries are given. In the case of constant flow velocity, the direct method (cf. [37-39]) is used to derive an infinite set of local conservation laws of the $G$-equation. The derived infinite sets of local symmetries and conservation laws are used to develop novel numerical schemes to perform calculations in practical applications involving the $G$-equation. 


\subsubsection{Hidden symmetries and reductions for ideal magnetohydrodynamics equilibria}

In [85], hidden symmetries are derived for the axially symmetric steady-state solutions of the ideal magnetohydrodynamic equations. A reduction of these equations to a scalar second-order partial differential equation is obtained. Applications of the hidden symmetries yield large families of exact axially symmetric MHD equilibria.

\subsection{Construction of conservation laws}

The direct method for constructing local conservation laws of PDEs is applicable to wide classes of PDE systems (cf. [37-39,42]). Within this method, one seeks multipliers such that the linear combination of the PDEs in a given PDE system with the multipliers will yield a divergence expression. After local conservation law multipliers are found, one constructs the fluxes of the corresponding conservation law.

\subsubsection{Computation of fluxes of conservation laws}

In the review paper [86], there is a discussion of various methods for flux computation, including a comparison of these methods as well as illustrations by examples. There is also a presentation of the implementation of these methods in symbolic software.

\subsection{Extending the spaces of admitted symmetries}

\subsubsection{Potential systems for PDEs having several conservation laws}

Knowledge of the symmetry properties of the modelling PDE system of a physical process can be very useful for understanding the behaviour of solutions, e.g., the group invariance property of a PDE system allows one to generate new solutions from known ones (cf. [85]), to construct conservation laws (cf. [86]), and find wide classes of exact invariant solutions. For many nonlinear systems, invariant solutions are the only known solutions and can be used as testing solutions for numerical and other approximate solutions. Hence knowledge of a new symmetry can be of great importance for a given PDE system. In [87], another way of constructing potential systems is illustrated that can lead to finding new potential symmetries (cf. [10,42,44,46,47,55]) of a given PDE system.

\subsection{Symmetry-classification algorithms}

Recent developments in solving overdetermined systems of differential equations and their implementation in terms of various symbolic software packages have allowed one to solve seemingly intractable problems of symmetry classification.

\subsubsection{An algorithm for the complete symmetry classification of differential equations based on Wu's method}

In [88], an algorithm is presented which gives a new application of Wu's method (differential characteristic set algorithm) for the complete symmetry classification of differential equations containing arbitrary parameters/functions. As illustrative examples, complete potential symmetry classifications of linear and nonlinear wave equations with an arbitrary function as well as classical and nonclassical symmetries of parametric Burgers' equations are presented. 


\subsubsection{Algorithmic symmetry classification with invariance}

In [89], symmetry classification for a system of differential equations is achieved algorithmically by applying a differential reduction and completion (DRC) algorithm (cf. [63]) to the linear infinitesimal determining equations of the system. It is shown that the invariance of the classification under the action of the equivalence group can be tested algorithmically knowing only the determining equations of the equivalence group. The method is implemented in Maple.

\subsection{Numerical methods involving symmetries and/or conservation laws}

\subsubsection{How to adaptively resolve evolutionary singularities in differential equations with symmetry}

In [90], the theory of self-similar blow-up in evolutionary differential equations is reviewed and a moving mesh method is presented to simulate numerically such phenomena. The method exploits the evolving symmetries in such problems to guide the adaptivity in both time and space. This is shown to provide an efficient and reliable way to simulate self-similar singularity formulation. This enables one to capture dynamics where the behaviour is exactly or asymptotically self-similar. It turns out that this method is simple to program and extends the utility of naive finite difference discretization methods. The focus is on the practical implementation with examples drawn from applications.

\subsubsection{A rarefaction-tracking method for hyperbolic conservation laws}

In [91], a numerical method is presented for one-dimensional scalar conservation laws that combines the method of characteristics, local similarity solutions, and particle management. The solution is approximated by local similarity solutions. While traditional approaches use shocks, the presented method uses rarefaction and compression waves. Although shocks are not explicitly tracked, they can be located accurately. The method is exhibited by numerical examples. Specific applications are outlined as well as extensions of the method.

\subsection{Other topics}

\subsubsection{Invariance and first integrals of continuous and discrete Hamiltonian equations}

In [92], there is consideration of the relationship between symmetries and first integrals (conservation laws) for both continuous and discrete Hamiltonian equations. It is shown that canonical Hamiltonian equations can be obtained through a variational principle from an action functional. The invariance properties of the functional are considered as is done in the Lagrangian formalism. The well-known Noether identity is rewritten in terms of the Hamiltonian and symmetry operators. This is shown to provide a simple and clear way to construct first integrals of Hamiltonian equations without integration. The discrete analog of this identity for discrete Hamiltonian equations can be used to construct conservative finite-difference schemes in the Hamiltonian framework that are important in numerical implementations.

\subsubsection{Coarsening dynamics of slipping droplets}

In [93], the late-phase dewetting process of nanoscopic thin polymer films on hydrophobized substrate is studied, using some recent lubrication models that take account of large slippage at the polymer-substrate interface. 


\subsubsection{Ultrasound detection of externally induced microthrombi cloud formation: a theoretical study}

In [94], a mathematical model is presented for the formation of microaggregates (microthrombi) of fibrin polymers in blood flow, induced by an external source.

\subsection{Open problems}

During the Vancouver conference, there was a two-part session, led by George Bluman, on open problems relating to the Similarity areas 2.1-2.6. The tape of the first part, which includes presentations on open problems by Andrew Bernoff, George Bluman, Philip Broadbridge, Chris Budd and Jean-François Ganghoffer, is available at the following website address: http://new.pims.math.ca/pix/video/similarity4.mov. Unfortunately, the tape of the shorter second part is unavailable.

\section{References}

1. Buckingham E (1914) On physically similar systems; illustrations of the use of dimension equations. Phys Rev 4:345-376

2. Buckingham E (1915) The principles of similitude. Nature 96:396-397

3. Buckingham E (1915) Model experiments and the form of empirical equations. Trans ASME 37:263-296

4. Bridgman PW (1931) Dimensional analysis, 2 edn. Yale University Press, New Haven

5. Barenblatt GI (1979) Similarity, self-similarity, and intermediate asymptotics. Consultants Bureau, New York, 218 pp

6. Barenblatt GI (1987) Dimensional analysis. Gordon and Breach, New York, $134 \mathrm{pp}$

7. Barenblatt GI (1996) Scaling, self-similarity, and intermediate asymptotics. Cambridge University Press, Cambridge, 386 pp

8. Sedov LI (1982) Similarity and dimensional methods in mechanics, 9th edn. Mir, Moscow, 424 pp

9. Bluman GW, Cole JD (1974) Similarity methods for differential equations. Springer, New York, $332 \mathrm{pp}$

10. Bluman GW, Kumei S (1989) Symmetries and differential equations. Springer, New York, 412 pp

11. Bluman GW, Anco SC (2002) Symmetry and integration methods for differential equations. Springer, New York, $419 \mathrm{pp}$

12. Giga Y, Kohn RV (1985) Asymptotically self-similar blowup of semilinear heat equations. Commun Pure Appl Math 38:297-319

13. Giga Y, Kohn RV (1987) Characterizing blowup using self-similar variables. Indiana Univ Math J 36:1-40

14. Levine HA (1989) Quenching, nonquenching, and beyond quenching for the solution of some parabolic equations. Ann Math Pure Appl 155:243-260

15. Levine HA (1990) The role of critical exponents in blowup theorems. SIAM Rev 32:262-288

16. Fillipas S, Kohn RV (1992) Refined asymptotics for the blowup of $u_{t}-\Delta u=u^{p}$. Commun Pure Appl Math 45:821-869

17. Galaktionov VA, Vázquez JL (2002) The problem of blow-up in nonlinear parabolic equations. Discret Contin Dyn Syst 8:399-433

18. Galaktionov VA, Williams JF (2004) On very singular similarity solutions of a higher order semilinear parabolic equation. Nonlinearity 17:1075-1099

19. Budd CJ, Galaktionov VA, Williams JF (2004) Self-similar blow-up in higher-order parabolic equations. SIAM J Appl Math 64:1775-1809 (electronic)

20. Evans JD, Galaktionov VA, King JR (2007) Source-type solutions of the fourth-order unstable thin film equation. Eur J Appl Math 18:1799-1841

21. Bernoff AJ, Bertozzi AL (1995) Singularities in a modified Kuramoto-Sivashinsky equation describing interface motion for phase transition. Physica D 85:375-404

22. Bernoff AJ, Bertozzi AL, Witelski TP (1998) Axisymmetric surface diffusion; dynamics and stability of self-similar pinchoff. J Stat Phys 93:725-776

23. Bertozzi AL, Pugh MC (2000) Finite-time blowup of solutions of some long-wave unstable thin film equations. Indiana Univ Math J 49:1323-1366

24. Herrero MA, Velázquez JJL (1996) Singularity formation in a chemotaxis model. Math Ann 306:583-623

25. Brenner MP, Constantin P, Kadanoff LP, Schenkel A, Venkataramani SC (1997) Diffusion, attraction, and collapse. Nonlinearity 10:1739-1754

26. Guo Y, Pan Z, Ward MJ (2006) Touchdown and pull-in voltage behavior of a MEMS device with varying dielectric properties. SIAM J Appl Math 66:309-338

27. Eggers J, Fontelos MA (2009) The role of self-similarity in singularities of partial differential equations. Nonlinearity 22:R1-R44

28. Bernoff AJ, Witelski TP (2010) Stability and dynamics of self-similarity in evolution equations. J Eng Math. doi:10.1007/ s10665-009-9309-8

29. Galaktionov VA, Vazquez JL (2004) A stability technique for evolution partial differential equations: a dynamical systems approach. Progress in nonlinear differential equations and their applications, vol 56. Birkhäuser, Boston, 377 pp 
30. Hocking LM, Stewartson K, Stuart JT (1972) A nonlinear instability burst in plane parallel flow. J Fluid Mech 51:705-735

31. Dupont TF, Goldstein RE, Kadanoff LP, Zhou SM (1993) Finite-time singularity formation in Hele-Shaw systems. Phys Rev E 47:4182-4196

32. Quittner O, Souplet P (2007) Superlinear parabolic problems: blow-up, global existence and steady states. Birkhäuser advanced texts. Birkhäuser, Basel, $684 \mathrm{pp}$

33. Olver PJ (1986) Applications of Lie groups to differential equations. Springer, New York, $497 \mathrm{pp}$

34. Bluman GW, Kumei S (1990) Symmetry-based algorithms to relate partial differential equations, I. local symmetries. Eur J Appl Math 1:189-216

35. Bluman GW, Kumei S (1990) Symmetry-based algorithms to relate partial differential equations, II: linearization by nonlocal symmetries. Eur J Appl Math 1:217-223

36. Mikhailov AV, Shabat AB, Sokolov VV (1991) The symmetry approach to classification of integrable equations. In: Zakharov VE (ed) What is integrability. Springer, Berlin, pp 115-184

37. Anco SC, Bluman GW (1997) Direct construction of conservation laws from field equations. Phys Rev Lett 78:2869-2873

38. Anco SC, Bluman GW (2002) Direct construction method for conservation laws of partial differential equations. Part I: examples of conservation law classifications. Eur J Appl Math 14:545-566

39. Anco SC, Bluman GW (2002) Direct construction method for conservation laws of partial differential equations. Part II: general treatment. Eur J Appl Math 14:567-585

40. Bluman GW, Temuerchaolu G, Anco SC (2006) New conservation laws obtained directly from symmetry action on a known conservation law. J Math Anal Appl 322: 233-250

41. Anco SC, Bluman GW, Wolf T (2008) Invertible mappings of nonlinear PDEs to linear PDEs through admitted conservation laws. Acta Appl Math 101:21-38

42. Bluman GW, Cheviakov AF, Anco SC (2009) Applications of symmetry methods to partial differential equations. Springer, New York

43. Olver PJ (1977) Evolution equations possessing infinitely many symmetries. J Math Phys 18:1212-1215

44. Bluman GW, Kumei S, Reid GJ (1988) New classes of symmetries for partial differential equations. J Math Phys 29:806-811

45. Akhatov IS, Gazizov RK, Ibragimov NH (1991) Nonlocal symmetries. Heuristic approach. J Sov Math 55:1401-1450

46. Bluman GW, Cheviakov AF, Ivanova NM (2006) Framework for nonlocally related partial differential equations systems and nonlocal symmetries: extension, simplification, and examples. J Math Phys 47:113505

47. Bluman GW, Cheviakov AF, Ganghoffer J-F (2008) Nonlocally related PDE systems for one-dimensional nonlinear elastodynamics. J Eng Math 62:203-221

48. Bluman GW, Cole JD (1969) The general similarity solution of the heat equation. J Math Mech 18:1025-1042

49. Clarkson PA, Kruskal MD (1989) New similarity solutions of the Boussinesq equation. J Math Phys 30:2201-2213

50. Levi D, Winternitz P (1989) Non-classical symmetry reduction: example of the Boussinesq equation. J Phys A 22:2915-2924

51. Burde GI (1996) New similarity reductions of the steady-state boundary layer equations. J Phys A 29:1665-1683

52. Martina L, Sheftel MB, Winternitz P (2001) Group foliation and non-invariant solutions of the heavenly equation. J Phys A 34:9243-9263

53. Bluman GW, Yan Z (2005) Nonclassical potential solutions of partial differential equations. Eur J Appl Math 16:239-261

54. Galaktionov VA, Svirshchevskii SR (2007) Exact solutions and invariant subspaces of nonlinear partial differential equations in mechanics and physics. Chapman and Hall, Boca Raton, 498 pp

55. Cheviakov AF (2008) An extended procedure for finding exact solutions of PDEs arising from potential symmetries. Applications to gas dynamics. J Math Phys 49:083502

56. Wu WT (1984) Basic principles of mechanical theorem proving in elementary geometries. J Syst Sci Math Sci 4:207-235

57. Kersten PHM (1987) Infinitesimal symmetries: a computational approach. CWI tract no. 34. Centrum voor Wiskunde en Informatica, Amsterdam, $155 \mathrm{pp}$

58. Reid GJ (1990) A triangularization algorithm which determines the Lie symmetry algebra of any system of PDEs. J Phys A 23:L853-L859

59. Reid GJ (1991) Algorithms for reducing a system of PDEs to standard form, determining the dimension of its solution space and calculating its Taylor series solution. Eur J Appl Math 2:293-318

60. Reid GJ (1991) Finding abstract symmetry algebra of differential equations of differential equations without integrating determining equations. Eur J Appl Math 2:319-340

61. Wolf T, Brand A (1992) The computer algebra package CRACK for investigating PDEs. In: Proceedings of ERCIM advanced course on partial differential equations and group theory, Bonn

62. Boulier F, Lazard D, Ollivier F, Petitot M (1995) Representation for the radical of a finitely generated differential ideal. In: ISAAC 95: proceedings of the 1995 international symposium on symbolic and algebraic computation. ACM, New York, pp 158-166

63. Reid GJ, Wittkopf AD, Boulton A (1996) Reduction of systems of nonlinear partial differential equations to simplified involutive forms. Eur J Appl Math 7:604-635

64. Mansfield EL (1996) The differential algebra package diffgrob2. Maple Tech 3:33-37

65. Hereman W (1997) Review of symbolic software for Lie symmetry analysis. Math Comput Model 25:115-132

66. Wolf T (2002) Investigating differential equations with CRACK. In: Grabmeier J, Kaltofen E, Weispfenning V (eds) LiePDE, Applysymm and ConLaw, handbook of computer algebra, foundations, applications, systems. Springer, New York, pp 465-468

67. Wolf T (2002) A comparison of four approaches to the calculation of conservation laws. Eur J Appl Math 13:129-152 
68. Temuerchaolu G (2003) An algorithmic theory of reduction of differential polynomial systems. Adv Math 32:208-220 (in Chinese)

69. Wittkopf AD (2004) Algorithms and implementations for differential elimination. PhD thesis, Simon Fraser University, Burnaby. http://ir.lib.sfu.ca:8080/retrieve/205/etd0400.pdf

70. Hereman W (2005) Symbolic computation of conservation laws of nonlinear partial differential equations in multi-dimensions. Int J Quantum Chem 106:278-299

71. Cheviakov AF (2007) GeM software package for computation of symmetries and conservation laws of differential equations. Comput Phys Commun 176:48-61

72. Budd CJ, Iserles A (1999) Geometrical integration: numerical solution of differential equations on manifolds. Philos Trans R Soc Lond Ser A 357:945-956

73. Dorodnitsyn VA (2000) Group properties of difference equations. MAKC Press, Moscow, 210 pp (in Russian)

74. Levi D, Winternitz P (2006) Continuous symmetries of difference equations. J Phys A 39:1-63

75. Berger M, Kohn RV (1988) A rescaling algorithm for the numerical calculation of blowing-up solutions. Commun Pure Appl Math 41:841-863

76. Witelski T (2001) Computing finite-time singularities in interfacial flows. In: Modern methods in scientific computing and applications (Montreal, QC, 2001). Nato Sc. Ser. II Math. Phys. Chem., vol 75. Kluwer, Dordrecht, pp 451-487

77. Budd CJ, Carretero-Gonzalez R, Russell RD (2005) Precise computations of chemotactic collapse using moving mesh methods. J Comput Phys 2002:463-487

78. Peschka D, Münch A, Niethammer B (2010) Thin-film rupture for large slip. J Eng Math. doi:10.1007/s10665-009-9342-7

79. Grunewald N, Levy R, Mata M, Ward T, Bertozzi AL (2010) Self-similarity in particle-laden flows at constant volume. J Eng Math. doi:10.1007/s10665-009-9345-4

80. Huppert HE (1982) Flow and instability of a viscous current down a slope. Nature 300:427-429

81. King JR (2010) Asymptotic analysis of extinction behaviour in fast nonlinear diffusion. J Eng Math. doi:10.1007/ s10665-009-9329-4

82. Broadbridge P, Goard JM (2010) Temperature-dependent surface diffusion near a grain boundary. J Eng Math. doi:10.1007/ s10665-009-9332-9

83. Ganghoffer J-F, Magnenet V, Rahouadj R (2010) Relevance of symmetry methods in mechanics of materials. J Eng Math. doi:10. 1007/s10665-009-9311-1

84. Oberlack M, Cheviakov AF (2010) Higher-order symmetries and conservation laws of the $G$-equation for premixed combustion and resulting numerical schemes. J Eng Math. doi:10.1007/s10665-009-9339-2

85. Bogoyavlenskij O (2010) Restricted Lie point symmetries and reductions for ideal magnetohydrodynamics equilibria. J Eng Math. doi:10.1007/s10665-009-9326-7

86. Cheviakov AF (2010) Computation of fluxes of conservation laws. J Eng Math. doi:10.1007/s10665-009-9307-x

87. Ivanova NM (2010) Potential systems for PDEs having several conservation laws. J Eng Math. doi:10.1007/s10665-009-9308-9

88. Chaolu T, Jing P (2010) An algorithm for the complete symmetry classification of differential equations based on Wu's method. J Eng Math. doi:10.1007/s10665-009-9344-5

89. Lisle I, Huang S-LT (2010) Algorithmic symmetry classification with invariance. J Eng Math. doi:10.1007/s10665-009-9327-6

90. Budd CJ, Williams JF (2010) How to adaptively resolve evolutionary singularities in differential equations with symmetry. J Eng Math. doi:10.1007/s10665-009-9343-6

91. Farjoun Y, Seibold B (2010) A rarefaction-tracking method for hyperbolic conservation laws. J Eng Math. doi:10.1007/ s10665-009-9338-3

92. Dorodnitsyn V, Kozlov R (2010) Invariance and first integrals of continuous and discrete Hamiltonian equations. J Eng Math. doi:10.1007/s10665-009-9312-0

93. Kitavtsev G, Wagner B (2010) Coarsening dynamics of slipping droplets. J Eng Math. doi:10.1007/s10665-009-9313-z

94. Guria GT, Herrero MA, Zlobina KE (2010) Ultrasound detection of externally induced microthrombi cloud formation: a theoretical study. J Eng Math. doi:10.1007/s10665-009-9340-9 